OPEN ACCESS

Edited by:

Lakshmanan Krishnamurti, Emory University, United States

Reviewed by:

Sushil Selvarajan, Christian Medical College \& Hospital, India Yann Lamarre, University of São Paulo, Brazi

*Correspondence: Olivier Hequet olivier.hequet@efs.sante.fr

Specialty section: This article was submitted to Hematology,

a section of the journa

Frontiers in Medicine

Received: 18 July 2021 Accepted: 29 October 2021 Published: 22 December 2021

Citation:

Hequet $O$, Boisson $C$, Joly $P$, Revesz D, Kebaill K, Gauthier A, Renoux C, Creppy S, Nader E, Nicolas JF, Berard F, Cognasse F,

Vocanson $M$, Bertrand $Y$ and Connes $P$ (2021) Priming With Red Blood Cells Allows Red Blood Cell Exchange for Sickle Cell Disease in Low-Weight Children. Front. Med. 8:743483. doi: 10.3389/fmed.2021.743483

\section{Priming With Red Blood Cells Allows Red Blood Cell Exchange for Sickle Cell Disease in Low-Weight Children}

\author{
Olivier Hequet ${ }^{1,2 *}$, Camille Boisson ${ }^{3,4,5}$, Philippe Joly ${ }^{3,4,5}$, Daniela Revesz ${ }^{1}$, \\ Kamila Kebaili ${ }^{3,4,6}$, Alexandra Gauthier ${ }^{3,4,6}$, Celine Renoux ${ }^{3,4,5}$, Severine Creppy ${ }^{7}$, \\ Elie Nader ${ }^{3,4}$, Jean François Nicolas ${ }^{2,8}$, Frédéric Berard ${ }^{2,8}$, Fabrice Cognasse $^{9}$, \\ Marc Vocanson ${ }^{2}$, Yves Bertrand ${ }^{6}$ and Philippe Connes ${ }^{3,4}$ \\ ${ }^{1}$ Etablissement Français du Sang Rhône Alpes, Apheresis Unit, Centre Hospitalier Lyon Sud, Lyon, France, ${ }^{2}$ CIRI, \\ International Center for Infectiology Research, INSERM U1111, Université de Lyon, Lyon, France, ${ }^{3}$ Laboratoire \\ Interuniversitaire de Biologie de la Motricité (LIBM) EA7424, Equipe "Biologie Vasculaire et du Globule Rouge", Université \\ Claude Bernard Lyon 1, Lyon, France, ${ }^{4}$ Laboratoire d'Excellence Sur le Globule Rouge (Labex GR-Ex), Paris, France, \\ ${ }^{5}$ Service de Biochimie et Biologie Moléculaire, Laboratoire de Biologie Médicale Multi-site, Hospices Civils de Lyon, Lyon, \\ France, ${ }^{6}$ Institut d'Hématologie et d'Oncologie Pédiatrique, Hospices Civils de Lyon, Lyon, France, ${ }^{7}$ Distribution Unit, Centre \\ Hospitalier Edouard Herriot, Etablissement Français du Sang Auvergne Rhône Alpes, Lyon, France, ${ }^{8}$ Clinical Immunology \\ and Allergology, Centre Hospitalier Lyon Sud, Hospices Civils de Lyon, Lyon, France, ${ }^{9}$ Scientific Department, Etablissement \\ Français du Sang Auvergne-Rhône-Alpes, Saint-Etienne, France
}

Red blood cell exchanges are frequently used to treat and prevent cerebrovascular complications in patients with sickle cell anemia (SCA). However, the low weight of young children represents serious concerns for this procedure. The Spectra Optia device can perform automatic priming using red blood cells (RBCs) (RCE/RBC-primed) which could allow RBC exchanges (RCE) to be performed in young children without hypovolemic complications, but this method requires evaluation. We prospectively analyzed the clinical safety of the RCE/RBC-primed procedure in 12 SCA low-weight children under either a chronic RCE program or emergency treatment over 65 sessions. We monitored grade 2 adverse events (AEs) such as a decrease in blood pressure, increase in heart rate, fainting sensation, or transfusion reactions and identified the critical times during the sessions in which AEs could occur. Post-apheresis hematocrit (Hct) and a fraction of cell remaining (FCR) values were compared to the expected values. We also compared the impact of automatic RCE ( $n=7)$ vs. RCE/RBC-primed $(n=8)$ on blood viscosity and RBC rheology. A low incidence of complications was observed in the 65 RCE sessions with only seven episodes of transient grade 2 AEs. Post-apheresis Hct and FCR reached expected values with the RCE/RBC-primed method. Both the automatic and priming procedures improved RBC deformability and decreased the sickling tendency during deoxygenation. Blood rheological features improved in both RCE/RBC-primed and automatic RCE without priming conditions. The RCE/RBC-primed procedure provides blood rheological benefits, and is safe and efficient to treat, notably in young children with SCA in prophylactic programs or curatively when a SCA complication occurs.

Keywords: red blood cell exchange, sickle cell anemia, low weight children, priming, safety, performances, blood rheology 


\section{INTRODUCTION}

Sickle cell anemia is caused by a point mutation in the seventh position of the $\beta$-globin gene leading to the production of abnormal hemoglobin called HbS. When deoxygenated, polymerization of $\mathrm{HbS}$ may occur, leading to mechanical distortion of red blood cells (RBC), i.e., sickling (1). Decreased deformability and increased fragility of sickled RBCs are the cause of frequent painful vaso-occlusive events and enhanced hemolysis in patients with sickle cell anemia (SCA), respectively $(1,2)$. Moreover, patients with the highest blood viscosity are at high risk for frequent vasoocclusive crises and other complications $(2,3)$. Recurrent vaso-occlusive events additionally cause endothelial dysfunction and inflammation, resulting in progressive tissue and organ damage (3-5).

Replacement of sickle RBCs containing HbS with healthy RBCs containing normal hemoglobin improves the outcome of patients with SCA. This replacement can be achieved by transfusions associated with manual bloodletting. However, this methodology allows a limited exchange of abnormal RBCs and may generate blood hyperviscosity, which in turn may result in vascular complications, and does not prevent iron overload (6). RBC exchange (RCE) using an apheresis device can replace a greater number of RBCs, is more efficient in preventing the occurrence of SCA complications when regularly performed, decreases whole blood viscosity, and limits the risk of iron overload (6-10).

However, the extracorporeal volume (EV) (160-185 ml depending on the device) of the RCE circuit limits its use in low-weight children (11-13). Transfusion experts suggest performing RCE without priming only in children with a bodyweight higher than $20 \mathrm{~kg}$ (with total blood volume [TBV] around $1,500 \mathrm{ml}$ and EV corresponding to $12 \%$ ) (14). When $\mathrm{EV}$ is greater than $15 \%$ of the total blood volume (TBV), they recommend performing blood priming before RCE (15). In addition to weight, hematocrit (Hct) must be considered when performing RCE since low weight associated with low Hct predisposes to hypovolemic complications during RCE (16).

A few years ago, the Cobe Spectra ${ }^{\circledR}$ device was replaced by Spectra Optia ${ }^{\circledR}$, which was the first device relying on a completely automatic priming procedure using $\mathrm{RBC}$ during RCE (RCE/RBC-primed). RCE/RBC-primed could allow RCE to be performed in young children without hypovolemic complications but this method requires evaluation. The main goal of this study was to assess the feasibility and safety of RCE/RBC-primed in low-weight children with SCA. In addition, we evaluated the impact of RCE/RBCprimed on RBC rheology and blood viscosity and compared

Abbreviations: RBC, Red blood cell; RCE, Red blood cell exchange; SCA, Sickle cell anemia; AE, Adverse events; Hct, Hematocrit; FCR, Fraction of cell remaining; EV, Extracorporeal volume; TBV, Total blood volume; sBP, systolic blood pressure; RBCU, Red blood cell units; aFCR, actual FCR; pFCR, predictive FCR; fFCR, final FCR; nHct, native Hct; pHct, predictive Hct; fHct, final Hct; PoS, Point of sickling; ACD-A, acid citrate dextrose A. the responses to the ones obtained with RCE without $\mathrm{RBC}$ priming.

\section{MATERIALS AND METHODS}

\section{Patient Selection}

This prospective study was conducted in accordance with the Declaration of Helsinki and approved by the Hospices Civils de Lyon ethics committee (L14-127). Twelve children with SCA were included after obtaining parental written informed consent. The inclusion criterion was a body weight $<20 \mathrm{~kg}$ and the exclusion criterion was hemodynamic instability with mean systolic blood pressure $(\mathrm{sBP})<50 \mathrm{mmHg}$.

\section{Description of the RCE With Personalized Priming Procedure}

In chronic programs, RCE sessions were performed every 6 weeks. Otherwise, RCE sessions were performed when an acute SCA complication occurred. Venous access was short-term central venous access in all cases.

Irregular agglutinin research was performed before RCE sessions. Transfused RBC units (RBCUs) were sickle-negative, leuco-reduced, crossmatch-compatible, and phenotypically matched for the C, E, c, e, and K (JK2 and MNS3 when possible) antigens. All the RBCUs transfused were heated to $37^{\circ} \mathrm{C}$ in a water bath.

Considering the low weight of the children, the TBV was calculated using the initial and simplified formulae of Glicher: TBV $(\mathrm{ml})=$ weight $(\mathrm{kg}) \times 75(17)$. The number of RBCUs needed can easily be deduced since the Spectra Optia ${ }^{\circledR}$ software (version 7.2) calculates the volume of transfused RBCs required to reach the predictive fraction of cell remaining (pFCR) and the predictive Hct (pHct). To perform RBC priming, a supplementary compatible RBCU (called reconstituted RBCU) was ordered and completed with ABO-compatible fresh frozen plasma to obtain a reconstituted RBCU. To prevent hemoconcentration during the critical session stage, i.e., after infusion of $185 \mathrm{ml}$ of concentrated RBCs while the device has not removed significant amounts of abnormal RBCs, we primed the circuit with RBCU diluted with $\mathrm{ABO}$-compatible fresh frozen plasma targeting an Hct close to the blood level of the patient, i.e., with an Hct of $28 \%$, based on our clinical experience.

After discontinuation of the priming circuit (volume $200 \mathrm{ml}$ and flow $60 \mathrm{ml} / \mathrm{min}$ ), the reconstituted $\mathrm{RBCU}$ was removed, and the RBC-primed circuit was connected to the central venous access. Depending on the degree of filling of the infusion chamber, the EV of the RCE for the Spectra Optia ${ }^{\circledR}$ (Terumo BCT) device circuit ranges from 141 to $185 \mathrm{ml}$ (14), and the latter value was chosen for further calculations. Since rinse-back is not recommended at the end of the RCE in children (15), $15 \mathrm{ml} / \mathrm{kg}$ of $4 \%$ albumin was continuously perfused to prevent hypovolemia at the end of the procedure.

Anticoagulation of the circuit was performed using acid citrate dextrose solution A (ACD-A), and a significant decrease in blood calcium concentration was prevented by continuous intravenous calcium infusion. 


\section{Clinical Tolerance, a Fraction of Cell Remaining, Hematocrit, and Factors Prone to Influence Hemostasis}

The clinical tolerance of RCE using RCE/RBC-primed in lowweight children was divided into three periods: during the first 10 min of the RCE/RBC-primed sessions, corresponding to the time between the blood intake of the patient and the infusion of normal RBCs (called the critical time of priming reinfusion), and during two consecutive 40-min periods. Systolic BP and heart rate (HR) were monitored every 2 min during the critical time of priming reinfusion and every $10 \mathrm{~min}$ during the remaining session. According to the National Cancer Institute Common Terminology Criteria for Adverse Events guidelines (18), grade 2 AEs or higher correspond to a significant decrease in sBP $(>20 \mathrm{mmHg}$ ) and a significant increase in HR ( $>20$ pulses per minute) associated or not with a significant fainting/asthenia sensation. The number of RCE/RBC-primed sessions with grade 2 AEs or higher were recorded, as well as the symptoms of transfusion-related AEs (pruritus, urticarial erythematous wheals, skin edema, and wheezing).

At the end of the RCE sessions, post-apheresis Hct was assessed to obtain actual Hct (aHct). In parallel, the postapheresis percentage of $\mathrm{HbS}$ (actual Hbs or aHbs) was assessed to calculate actual FCR (aFCR). The aFCR was calculated as follows: $\mathrm{aFCR}=\mathrm{aHbS}$ )/pre-apheresis $\mathrm{HbS}$ (also called native $\mathrm{HbS}$ or $\mathrm{nHbS}$ ). We also noted the predictive FCR (pFCR) which is recorded on the device before the sessions and the final FCR (fFCR), which corresponds to the FCR recorded by the device at the end of the sessions. In parallel, we noted pre-apheresis or native Hct (nHct), predictive Hct (pHct, recorded on the device before the session), and final Hct (fHct, recorded on the device at the end of the sessions).

Changes in factors prone to influence hemostasis parameters were assessed, i.e., ratio of $\mathrm{ACD}-\mathrm{A} /$ calcium infused and decrease in platelet blood levels (calculated as follows: decrease in platelet level $=[$ pre-apheresis blood level - post-apheresis blood level]/pre-apheresis blood level).

\section{Blood Rheology}

We also analyzed the impact of RBC-priming by comparing RCE/RBC-primed ( $n=8$ sessions in 5 of the 12 patients followed) vs. automatic RCE (i.e., without priming RBCs; $n$ $=7$ sessions in 7 other patients) on blood viscosity and RBC rheology. The two groups of patients were assessed during RCE programs. Measurements were performed before RCE/RBCprimed, at the end of the critical time of priming reinfusion (1015 first min) and the end of the sessions (Figure 1A). The same blood rheological parameters were measured at the same time points in patients having the RCE/RBC-primed and automatic classical RCE. Blood viscosity was measured at native Hct and shear rates of 45 and $90 \mathrm{~s}^{-1}$ using a cone-plate viscometer (Brookfield DVII+ with CPE40 spindle, Brookfield Engineering Labs, Natick, MA, USA) and expressed in centipoises (cP). RBC deformability was determined at $37^{\circ} \mathrm{C}$, in isotonic conditions and $3 \mathrm{~Pa}$ by laser diffraction analysis (ektacytometry), using the Laser-assisted Optical Rotational Cell Analyzer (LORRCA
Maxsis, RR Mechatronics, Hoorn, The Netherlands). In addition, ektacytometry was carried out with the oxygenscan module (LORRCA Maxsis, RR Mechatronics, Hoorn, The Netherlands) to measure $\mathrm{RBC}$ deformability over an oxygen gradient as previously described (19-22). The suspension was sheared at $30 \mathrm{~Pa}$ and $37^{\circ} \mathrm{C}$ into the Couette system of the ektacytometer. The oxygen partial pressure $\left(\mathrm{pO}_{2}\right)$ was gradually decreased from 160 to $20 \mathrm{mmHg}$ (deoxygenation) and then increased to normoxic values. The diffraction pattern obtained by ektacytometry was analyzed with a camera and a computer and the elongation index (EI), which reflects RBC deformability, was also calculated. The Point of Sickling ( $\mathrm{PoS})$ was obtained during deoxygenation and corresponds to the $\mathrm{pO}_{2}$ at which $\mathrm{RBC}$ deformability decreases below $5 \%$ of the maximal deformability reached during normoxia (i.e., before deoxygenation). All measurements were standardized as recommended $(21,22)$. RBC aggregation was determined at $37^{\circ} \mathrm{C}$ via syllectometry, (i.e., laser backscatter vs. time, using the LORRCA) after adjustment of the Hct to $40 \%$.

\section{Statistical Analyses}

Values are expressed as mean $\pm S D$. A one-way ANOVA with repeated measurements was used to compare aHct, pHct, and fHCT; aFCR, pFCR, and FFCR. A two way-ANOVA was used to compare the effects of RCE/RBC-primed and RCE without RBC priming on blood rheology. The Tukey post-hoc test was used to locate differences when appropriate. $p<0.05$ was considered significant.

\section{RESULTS}

\section{Feasibility and Safety of the Priming Protocol and the Whole RCE}

Twelve children were treated with RCE/RBC-primed (Table 1). In most children (10/12), EV of the circuit represented a high volume related to their TBV (EV/TBV ratio greater than 15\%) while lower EV/TBV was associated with low nHct (21 and 22\% for patients 6 and 12, respectively) were found in two of them.

During all sessions, it took approximately ten min ( 9 to $15 \mathrm{~min}$ ) for the device to reinfuse the volume of RBC-primed while the blood of the patient was still being removed. No significant grade 2 AEs (no decrease in sBP, no increase in HR, no fainting sensation) were observed during that time in the 65 RCE/RBC-primed sessions performed (Table 2).

The mean pFCR was $19 \pm 4 \%$ (Table 3), which corresponded to a significant RBC replacement $(68 \pm 8 \mathrm{ml} / \mathrm{kg}$ ) of at least $80 \%$ of the initial RBC volume. As recommended (15), the target Hct after RCEs in children was expected to be $27-30 \%$. Mean Hct levels in children after RCEs (aHct) were not significantly higher than $\mathrm{nHct}(27 \pm 2$ vs. $26 \pm 3 \%$, respectively), reducing the risk for developing hemodynamic complications during and after RCEs (Table 3).

The exchange step lasted $80 \pm 11 \mathrm{~min}$. All complications occurred during the last $30 \mathrm{~min}$ or in the first $5 \mathrm{~min}$ after the end of the session (Table 2). Systolic blood pressure (sBP) decreased in two patients just after the end of the sessions (Table 2). One episode of a significant increase in heart rate occurred in two patients and a fainting sensation occurred during three sessions 
RCE-RBC-primed

RCE without RBC priming

A

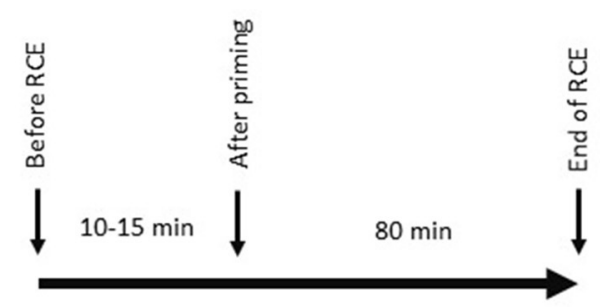

Blood rheological assesment
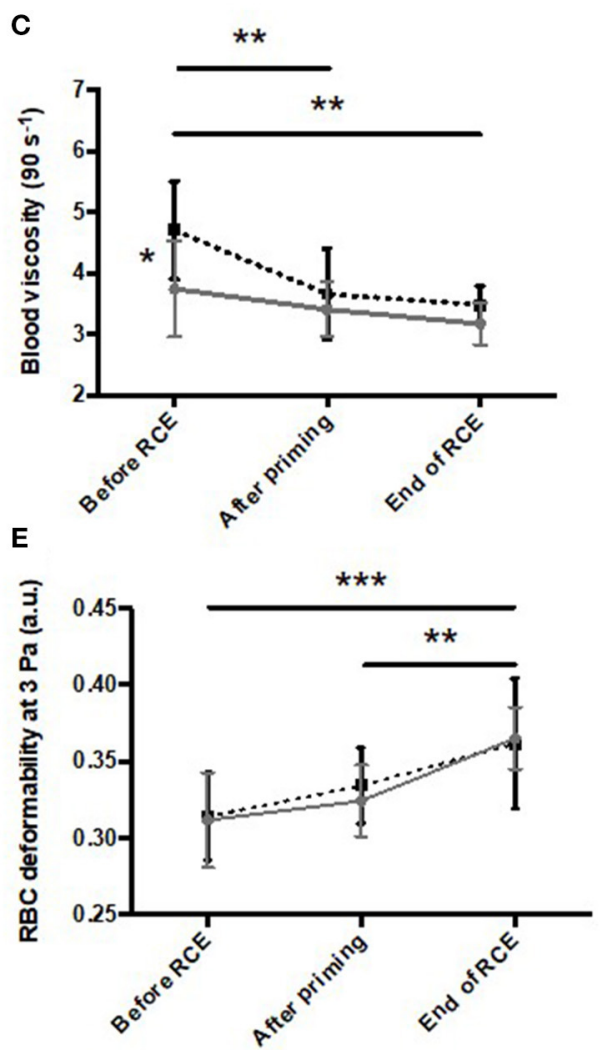

B

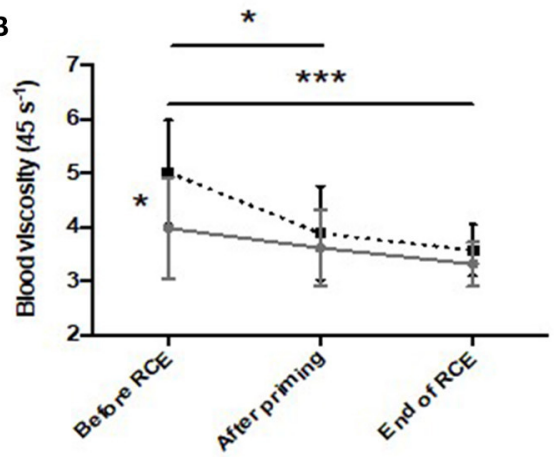

D

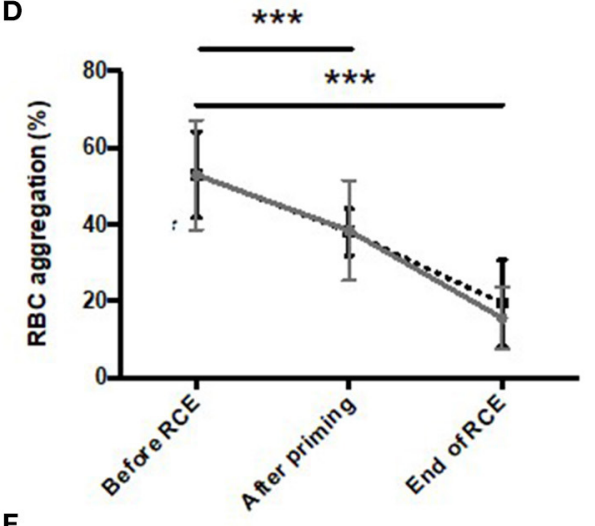

$\mathbf{F}$

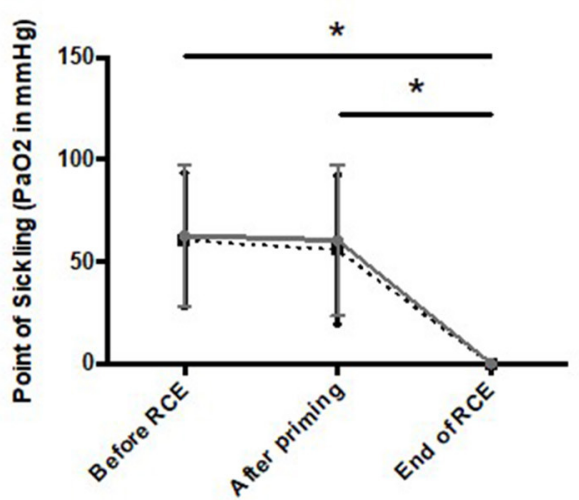

FIGURE 1 | Changes in blood rheological properties during red blood cell exchange/red blood cell (RCE/RBC)-primed vs. RCE without priming. (A) protocol; (B) blood viscosity at $45 \mathrm{~s}^{-1}$; (C) blood viscosity at $90 \mathrm{~s}^{-1}$; (D) RBC aggregation; (E) RBC deformability at $3 \mathrm{~Pa}$; (F) Point of sickling. Statistical difference: ${ }^{*} p<0.05$; ${ }^{\star *} p<0.01 ;{ }^{* \star *} p<0.001$.

in two children, all during the last $30 \mathrm{~min}$. Saline and $4 \%$ albumin were infused, inducing a decrease in HR or fainting sensation (while RCE continued) and a rapid increase in sBP. All the hemodynamic complications occurred at the end of the RCE sessions. The occurrence of AEs at this time suggested a role of the large volume of RBC exchanged (more than $80 \%$ of $\mathrm{RBC}$ exchanged i.e., aFCR $<20 \%$ ) and we could speculate that exchanging lower amounts of RBC may decrease the incidence of AEs. No pruritus or urticarial wheals and no wheezing were observed thereafter during the entire protocol (Table 2).

\section{Performances of RCE/RBC-Primed}

We analyzed the ability of the RCE/RBC-primed method to obtain the Hct required, and the ability to replace sufficient amounts of RBC in the blood of patients. No difference was observed between aHct, 
TABLE 1 | Clinical characteristics of the 12 patients with sickle cell anemia (SCA) at the beginning of red blood cell exchange/red blood cell (RCE/RBC)-primed.

\begin{tabular}{|c|c|c|c|c|c|c|c|c|c|c|c|c|}
\hline Parameters & Pt 1 & Pt 2 & Pt 3 & Pt 4 & Pt 5 & Pt 6 & Pt 7 & Pt 8 & Pt 9 & Pt 10 & Pt 11 & Pt 12 \\
\hline Gender & $\mathrm{F}$ & $\mathrm{F}$ & $\mathrm{F}$ & $\mathrm{M}$ & $\mathrm{M}$ & $\mathrm{M}$ & $\mathrm{F}$ & $M$ & $\mathrm{~F}$ & $\mathrm{M}$ & $\mathrm{F}$ & $\mathrm{F}$ \\
\hline Age (year) & 3 & 3.5 & 3.5 & 3 & 3 & 6 & 3 & 4.5 & 4.5 & 3 & 5.5 & 6 \\
\hline Weight (kg) & 11 & 15 & 13 & 12 & 13 & 18 & 13 & 16 & 17 & 13 & 17 & 19 \\
\hline TBV (mL) & 825 & 1,050 & 975 & 900 & 975 & 1,350 & 975 & 1,200 & 1,190 & 952 & 1,270 & 1,425 \\
\hline EC/TBV (\%) & 22 & 17 & 19 & 20 & 19 & 14 & 19 & 15 & 16 & 19 & 15 & 13 \\
\hline nHct (\%) & 28 & 31 & 21 & 28 & 26 & 21 & 22 & 23 & 12 & 30 & 22 & 22 \\
\hline RCE Program & Yes & yes & yes & no & yes & yes & yes & no & no & no & no & yes \\
\hline Indications for RCE & $\begin{array}{l}\text { ACS+ } \\
\text { Stroke }\end{array}$ & ACS & $A C S+V O C$ & ACS & $\mathrm{ACS}+\mathrm{VOC}$ & $\begin{array}{l}\text { CVO+ } \\
\text { Surgery }\end{array}$ & Stroke & ACS & Meningitis & Surgery & Surgery & $\begin{array}{c}\text { Cerebral } \\
\text { vasculopathy }\end{array}$ \\
\hline $\mathrm{nHbS}(\%)$ & 31 & 46 & 84 & 81 & 83 & 75 & 88 & 84 & 92 & 61 & 87 & 91 \\
\hline
\end{tabular}

Pt, patient; F, female; M, male; TBV, Total blood volume; VOC, vaso-occlusive crisis; ACS, acute chest syndrome; RBCP, packed red blood cells; RCE, red blood cell exchange; nHct, native hematocrit (blood hematocrit before RCE); $\mathrm{HH}$ bs, native HbS (blood HbS before RCE).

TABLE 2 | Clinical complications during RCE/RBC-primed.

\begin{tabular}{|c|c|c|c|c|c|c|c|c|c|c|c|c|c|}
\hline Parameters & Pt 1 & Pt 2 & Pt 3 & Pt 4 & Pt 5 & Pt 6 & Pt 7 & Pt 8 & Pt 9 & Pt 10 & Pt 11 & Pt 12 & All pts \\
\hline Number of RCE/RBC-primed sessions & 28 & 10 & 8 & 2 & 5 & 3 & 4 & 1 & 1 & 1 & 1 & 1 & 65 \\
\hline \multicolumn{14}{|l|}{ Decrease in sBPS } \\
\hline $2^{\text {nd }}$ period (11-50 min) & 0 & 0 & 0 & 0 & 0 & 0 & 0 & 0 & 0 & 0 & 0 & 0 & 0 \\
\hline $3^{\text {rd }}$ period (51-90 min) & $1 \mathrm{epd}^{*}$ & 0 & 0 & 0 & $1 \mathrm{epd}^{*}$ & 0 & 0 & 0 & 0 & 0 & 0 & 0 & 2 \\
\hline $2^{\text {nd }}$ period (11-50 min) & 0 & 0 & 0 & 0 & 0 & 0 & 0 & 0 & 0 & 0 & 0 & 0 & 0 \\
\hline $3^{\text {rd }}$ period (51-90 min) & 0 & 1 epd & 0 & 0 & 1 epd & 0 & 0 & 0 & 0 & 0 & 0 & 0 & 2 \\
\hline \multicolumn{14}{|l|}{ Fainting sensation } \\
\hline $1^{\text {st }}$ period $(0-10 \mathrm{~min})$ & 0 & 0 & 0 & 0 & 0 & 0 & 0 & 0 & 0 & 0 & 0 & 0 & 0 \\
\hline $2^{\text {nd }}$ period $(11-50 \mathrm{~min})$ & 0 & 0 & 0 & 0 & 0 & 0 & 0 & 0 & 0 & 0 & 0 & 0 & 0 \\
\hline
\end{tabular}

Pt, patient; sBP, systolic blood pressure; epd, episode. *occurrence during 5 min after the end of the sessions.

pHct, and fHct, or aFCR, pFCR, and fFCR (Table 3), indicating that the expected $\mathrm{Hct}$ and FCR values are reached.

The ratio ACD-A/calcium infused was around 10 in most of the patients (Table 3), as observed in our experience in adults treated with RCE (data not shown). The mean decrease in platelet levels was $61 \pm 13 \%$, which needs to be considered in some situations when RCE is performed.

\section{Blood Viscosity and RBC Rheology: Comparison of RCE/RBC-Primed vs. RCE Without Priming}

The use of an RBCU during the priming raised the question of transient blood hyperviscosity during the priming and at the end of the sessions that could promote complications in SCA. Indeed, blood rheological parameters were investigated in 8 young low-weight children with SCA ( $19 \pm 1 \mathrm{~kg} ; 4 \pm 1.2$ years) having the $\mathrm{RCE} / \mathrm{RBC}$-primed procedure and compared to the blood rheological responses of seven older children with SCA (33 $\pm 13 \mathrm{~kg} ; 12.4 \pm 3.6$ years) who were having the classical automatic RCE procedure without RBC-priming (Figure 1). Of note, the children of both groups were under chronic RCE programs and none of the sessions assessed was performed in an emergency.

Initial blood viscosity was lower in the RCE-RBC-primed group compared to the group having RCE without RBC priming. Blood viscosity remained unchanged in the patients having RCE/RBC-primed (Figures 1B,C). However, during automatic (classic) RCE, blood viscosity decreased after the first RBCU to reach similar blood viscosity to the other group up until the end of the procedure. RBC deformability increased and RBC aggregation decreased in the two groups over the procedures, with no difference between them (Figures 1D,E). The PoS decreased in the two groups to reach very low values at the end of the procedure and no difference was observed between the two groups (Figure 1F). 
TABLE 3 | Biological results to assess performances of RCE/RBC-primed.

\begin{tabular}{|c|c|c|c|c|c|c|c|c|c|c|c|c|c|}
\hline Parameters & Pt 1 & Pt 2 & Pt 3 & Pt 4 & Pt 5 & Pt 6 & Pt 7 & Pt 8 & Pt 9 & Pt 10 & Pt 11 & Pt 12 & All pts \\
\hline nHct (\%) & $28 \pm 1$ & $25 \pm 3$ & $22 \pm 1$ & $26 \pm 2$ & $26 \pm 3$ & $26 \pm 5$ & $23 \pm 1$ & 23 & 12 & 30 & 30 & 12 & $26 \pm 3$ \\
\hline pHct (\%) & $27 \pm 1$ & $27 \pm 1$ & $26 \pm 1$ & $27 \pm 1$ & $27 \pm 1$ & $27 \pm 1$ & $26 \pm 1$ & 28 & 25 & 29 & 28 & 26 & $27 \pm 1$ \\
\hline fHct (\%) & $28 \pm 1$ & $27 \pm 5$ & $25 \pm 1$ & $26 \pm 2$ & $27 \pm 1$ & $29 \pm 1$ & $26 \pm 1$ & 25 & 25 & 29 & 30 & 26 & $27 \pm 1$ \\
\hline aHct (\%) & $27 \pm 2$ & $28 \pm 3$ & $26 \pm 2$ & $27 \pm 1$ & $28 \pm 1$ & $29 \pm 1$ & $25 \pm 1$ & 25 & 26 & 29 & 29 & 26 & $27 \pm 2$ \\
\hline pFCR (\%) & $17 \pm 4$ & $18 \pm 3$ & $19 \pm 5$ & $17 \pm 3$ & $18 \pm 3$ & $20 \pm 0$ & $21 \pm 2$ & 20 & 20 & 20 & 18 & 20 & $19 \pm 4$ \\
\hline fFCR (\%) & $16 \pm 4$ & $16 \pm 2$ & $17 \pm 7$ & $17 \pm 4$ & $17 \pm 5$ & $19 \pm 0$ & $23 \pm 2$ & 20 & 20 & 20 & 19 & 26 & $18 \pm 5$ \\
\hline aFCR (\%) & $14 \pm 6$ & $17 \pm 4$ & $19 \pm 7$ & $12 \pm 2$ & $15 \pm 6$ & $17 \pm 6$ & $23 \pm 4$ & 19 & 23 & 19 & 18 & 22 & $17 \pm 6$ \\
\hline Number of RBCUs/session & 3 to 4 & 3 & 2 to 3 & 3 & 2 to 3 & 3 to 4 & 2 to 3 & 2 & 3 & 2 & 3 & 3 & 2 to 4 \\
\hline ACD-A infused to Pt (mL) & $116 \pm 22$ & $101 \pm 8$ & $94 \pm 18$ & $91 \pm 10$ & $99 \pm 11$ & $108 \pm 12$ & $87 \pm 19$ & 124 & 44 & 88 & 103 & 152 & $106 \pm 22$ \\
\hline Calcium injected (mL) & $11 \pm 3$ & $13 \pm 3$ & $10 \pm 3$ & $9 \pm 0$ & $10 \pm 3$ & $15 \pm 3$ & $10 \pm 2$ & 11 & 9 & 11 & 16 & 15 & $11 \pm 3$ \\
\hline Ratio ACD-A/calcium used & $11 \pm 2$ & $9 \pm 2$ & $10 \pm 1$ & $11 \pm 1$ & $10 \pm 3$ & $7 \pm 1$ & $8 \pm 1$ & 11 & 5 & 8 & 6 & 10 & $10 \pm 2$ \\
\hline PBL before RCE/RBC-primed & $183 \pm 62$ & $252 \pm 62$ & $304 \pm 50$ & $432 \pm 179$ & $297 \pm 98$ & $394 \pm 154$ & $398 \pm 53$ & 151 & 186 & 269 & 353 & 304 & $277 \pm 103$ \\
\hline PBL after RCE/RBC-primed & $79 \pm 13$ & $79 \pm 24$ & $75 \pm 12$ & $92 \pm 15$ & $54 \pm 27$ & $210 \pm 57$ & $152 \pm 48$ & 69 & 106 & 119 & 150 & 167 & $97 \pm 46$ \\
\hline Decrease in PBL (\%) & $52 \pm 10$ & $69 \pm 11$ & $76 \pm 4$ & $76 \pm 14$ & $79 \pm 3$ & $56 \pm 15$ & $61 \pm 14$ & 54 & 43 & 56 & 57 & 45 & $61 \pm 13$ \\
\hline
\end{tabular}

nHct, native (pre-apheresis Hct); pHCt, predictive Hct (expected Hct registered on device before session); fHct, post-apheresis Hct given by device after session; aHct, actual Hct (post-

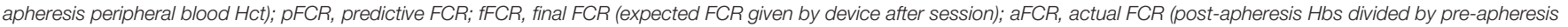
HbS); Pt, patient; PBL, platelet blood levels.

\section{DISCUSSION}

The indications of RCE during curative and prophylactic treatments of SCA are well-known (23). The principle of RCE is to prevent the occurrence of SCA complications by replacing abnormal RBCs with normal RBCs so that the HbS level does not exceed a defined threshold between two sessions $(15,23)$. Since prophylactic treatment has become more routine among apheresis teams who treat more and more Patients with SCA, the RCE method has to be improved to optimize this treatment in all patients, including the youngest ones. In very young children, two main issues hamper the use of RCE on a routine basis: 1) the venous access series [this is why we used temporary central venous catheter access for which we demonstrated tolerance and efficiency (24)] and 2) the risk of hypovolemia. The availability of a device combining RCE and an automatic priming step (RCE/RBC-primed) led us to evaluate its feasibility, safety, and performance in this population.

Our work showed that the RCE/RBC-primed method was well tolerated both during the priming and during the RCE step itself. The incidence of complications was thus very low and rapidly self-limiting. The method was safe without any risk of increasing blood viscosity during and after the sessions. Moreover, the $\mathrm{RCE} / \mathrm{RBC}$-primed method was efficient. i.e., allowed to reach the targeted post-apheresis FCR and Hct.

The main problem when performing RCE in very young children is the risk of hypovolemia due to a high EV compared to a low TBV. Indeed, the study of Dedeken et al. switched from manual exchange transfusion to RCE (using the Optia Spectra system) only in children who weighed $30 \mathrm{~kg}$ or more, this threshold having been decided to avoid priming of the circuit (7). RCE techniques with low EV, such as COBE Spectra, were used in low-weight children (20 to $30 \mathrm{~kg}$ ) without inducing hemodynamic complications $(25,26)$. In other apheresis techniques, RBC-priming appears to be a therapeutic option to treat children with a bodyweight below $20 \mathrm{~kg}$ (27). As mentioned, in our series the EV represented around $20 \%$ of the TBV of each child, which could induce severe hypovolemia and hemodynamic complications. Monitoring of the early phase of RCE while infusing the volume in the circuit primed with reconstituted RBCs showed no hemodynamic changes during initial blood withdrawal. This absence of early hemodynamic complications leads us to suggest that RBC-priming may be used more systematically in low-weight children. The complications that occurred in $10 \%$ of the sessions involved only hemodynamic events. Since they occurred at the end of the sessions, they were probably the consequence of extended $\mathrm{RBC}$ exchanges. Moreover, the occurrence of hemodynamic complications led us to consider the use of a continuous albumin infusion during RCE. However, this procedure with RBC-priming seemed to be necessary, as the frequency of AEs would probably be higher without RBC-priming in these low-weight children (14).

The performances of all types of RCE sessions, and here of $\mathrm{RCE} / \mathrm{RBC}$, had to be assessed to verify the efficiency and safety of this treatment in low-weight children. The main objective of RCE is to replace abnormal SCA-RBC with non-sickle RBC, which was evaluated by the FCR; the lower the FCR, the higher the replacement of SCA-RBC. Our results showed that the RCE/RBC-primed procedure was efficient since fFCR was similar to aFCR. Moreover, aHct was similar to both fHct and pHCt, excluding an increased risk for post-RCE stroke. Manual RBC exchange can cause a rise in Hct without improvement in blood viscosity $(10,28)$. The history of the first patient of this series summarizes the outcome and the rheological conditions after transfusion or manual RBC exchange and RCE. Within $24 \mathrm{~h}$ after a transfusion for ACS, this child developed a stroke and was successfully treated by RCE. Single transfusion may increase Hct and blood viscosity and cause vaso-occlusive complications $(28$, 
29). More recently it has been clearly shown that automated RCE and manual exchange display opposite changes in post-exchange Hct and blood viscosity (10). Manual exchanges can cause a rise in Hct without any improvement in blood viscosity while automated RCE decreases blood viscosity without any increase in Hct (10). As we added RBC during the infusion of primed-RBCs, we analyzed various parameters of blood rheology during and at the end of RCE, showing no increase in blood viscosity at the beginning of the session and a similar decrease in blood viscosity both in RCE/RBC-priming and RCE without RBC priming. The increase in $\mathrm{RBC}$ deformability, the decrease of the tendency of $\mathrm{RBC}$ to sickle (normal RBCs do not sickle), and the decrease in $\mathrm{RBC}$ aggregation during both RCE procedures may explain why blood viscosity decreased in the two groups, demonstrating no risk of blood hyperviscosity for the priming step.

Other factors need to be analyzed, in particular, hemostasis parameters as another blood product is transfused; this priming could influence the blood changes after sessions. Additional analysis of biological parameters showed a $60 \%$ decrease in platelet levels after RCE/RBC-priming. This decrease was in accordance with previous studies investigating the effects of RCE $(30,31)$. However, particular caution must be paid when performing RCE in patients with very recent stroke or at risk of bleeding, because of liver failure for example. In this last clinical situation, ACD-A infusion must be decreased or discontinued (32). In other cases, ACD-A infusion induces a decrease in calcium blood levels, which needs to be counteracted by calcium infusion. This calcium supplementation is particularly necessary for RCE in which ACD-A infusion is significant. The ratio between ACD-A and calcium (both infused) must be monitored, particularly in low-weight children. Even if no guidelines have yet been developed to consider the usage of calcium according to ACD-A, the ratio between both infusions must be monitored to prevent a bleeding risk associated with a decrease in platelets, especially when RCE is performed after stroke. In our study, the ratio ACD-A infused/calcium infused was monitored and was in

\section{REFERENCES}

1. Rees DC, Williams TN, Gladwin MT. Sickle-cell disease. Lancet. (2010) 376:2018-31. doi: 10.1016/S0140-6736(10)61029-X

2. Connes P, Renoux C, Romana M, Abkarian M, Joly P, Martin C, et al. Blood rheological abnormalities in sickle cell anemia. Clin Hemorheol Microcirc. (2018) 68:165-72. doi: 10.3233/CH-189005

3. Connes P, Lamarre Y, Waltz X, Ballas SK, Lemonne N, Etienne-Julan M, et al. Haemolysis and abnormal haemorheology in sickle cell anaemia. $\mathrm{Br} \mathrm{J}$ Haematol. (2014) 165:564-72. doi: 10.1111/bjh.12786

4. Conran N, Belcher JD. Inflammation in sickle cell disease. Clin Hemorheol Microcirc. (2018) 68:263-99. doi: 10.3233/CH-189012

5. Ballas SK. Sickle cell disease: Classification of clinical complications and approaches to preventive and therapeutic management. Clin Hemorheol Microcirc. (2018) 68:105-28. doi: 10.3233/CH-189002

6. Fasano RM, Meong T, Kaushal M, Sagiv E, Luban NLC, Riehm Meier E. Effectiveness of red blood cell exchange, partial manual exchange, and simple transfusion concurrently with iron chelation therapy in reducing iron overload in chronically transfused sickle cell anemia patients. Transfusion. (2016) 56:1707-15. doi: 10.1111/trf.13558 the same range in all sessions. In our experience in the field of $\mathrm{RCE}$, the ACD-A/calcium ratio of 10 prevents the symptoms of hypocalcemia and the bleeding risk (data not shown).

In conclusion, the usage of RBC-priming in low-weight children appears necessary and safe when performing RCE. RBC-priming did not modify the performances of RCE. Altogether, these results should encourage apheresis and pediatric hematological teams to perform RCE in these lowweight children while considering many precautions. In patients with higher body weight $(20-30 \mathrm{~kg})$ or low body weight (15$20 \mathrm{~kg}$ ) but low Hct, another possibility would be to perform albumin-priming. However, this therapeutic possibility has yet to be evaluated.

\section{DATA AVAILABILITY STATEMENT}

The raw data supporting the conclusions of this article will be made available by the authors, without undue reservation.

\section{ETHICS STATEMENT}

The studies involving human participants were reviewed and approved by Hospices Civils de Lyon Ethics Commitee (L14127). Written informed consent to participate in this study was provided by the participants' legal guardian/next of kin.

\section{AUTHOR CONTRIBUTIONS}

All authors listed have made a substantial, direct, and intellectual contribution to the work and approved it for publication.

\section{ACKNOWLEDGMENTS}

The authors thank the Association Pour la Recherche en Pathologie sur les Cellules Sanguines (ARPCS) for its funding for the acquisition of a cone plane viscosimeter.

7. Dedeken L, Lê PH, Rozen L, El Kenz H, Huybrechts S, Devalck C, et al. Automated RBC exchange compared to manual exchange transfusion for children with sickle cell disease is cost-effective and reduces iron overload. Transfusion. (2018) 58:1356-62. doi: 10.1111/trf.14575

8. Duclos C, Merlin E, Paillard C, Thuret I, Demeocq F, Michel G, et al. Long-term red blood cell exchange in children with sickle cell disease: manual or automatic? Transf Aph Sci. (2013) 48:21922. doi: 10.1016/j.transci.2012.09.002

9. Kuo KH, Ward R, Kaya B, Howard J, Telfer P. A comparison of chronic manual and automated red blood cell exchange transfusion in sickle cell disease patients. Br J Haematol. (2015) 170:425-8. doi: 10.1111/bjh. 13294

10. Ait Abdallah N, Connes P, Di Liberto G, Offredo L, Beaumont JL, Menouche $\mathrm{D}$, et al. Automated RBC exchange has a greater effect on whole blood viscosity than manual whole blood exchange in adult patients with sickle cell disease. Vox Sanguinis. (2020) 115:722-8. doi: 10.1111/vox.12990

11. Pratx LB, Gru I, Spotti M, Díaz Sánchez D, Ninomiya M, Marcarián $\mathrm{G}$, et al. Development of apheresis techniques and equipment designed for patients weighing less than $10 \mathrm{~kg}$. Transf Aph Sci. (2018) 57:3316. doi: $10.1016 / \mathrm{j}$.transci.2018.05.014 
12. Kim HC. Therapeutic pediatric apheresis. J Clin Aph. (2000) 15:12957. doi: 10.1002/(SICI)1098-1101(2000)15:1/2<129::AID-JCA7>3.0.CO;2-H

13. Golsdtein SL. Therapeutic apheresis in children: special considerations in children. Seminar Dial. (2012) 25:16570. doi: 10.1111/j.1525-139X.2011.01028.x

14. Perseghin P, Incontri A, Capra M, Pichler H, Witt V. Erythrocyte-exchange with the Optia ${ }^{\mathrm{TM}}$ cell separators in patients with sickle-cell disease. Transf Aph Sci. (2013) 28:411-5. doi: 10.1002/jca.21293

15. Sarode R, Ballas SK, Garcia A, Kim HC, King K, Sachais B, et al. Red blood cell exchange: 2015 American Society for Apheresis consensus conference on the management of patients with sickle cell disease. J Clin Apher. (2016) 32:342-67. doi: 10.1002/jca.21511

16. Merlin E, Hequet $\mathrm{O}$, Kanold J. Red blood cell exchange in children and adolescents with sickle cell disease. Transf Apher Sci. (2019) 58:13641. doi: 10.1016/j.transci.2019.03.006

17. Muraki R, Hirakoa A, Nagata T, Nakajima K, Oshita T, Arimichi M, et al. Novel method for estimating the total blood volume: the importance of adjustment using the ideal body weight and age for the accurate prediction of haemodilution during cardiopulmonary bypass. Inter Cardiovasc Thor Sur. (2018) 27:802-7. doi: 10.1093/icvts/ivy173

18. National Cancer Institute, National Institutes of Health U.S. Department of Health and Human Services. Common Terminology Criteria for Adverse Events (CTCAE) Version 4.0. NIH publication \# 09-7473. Revised Version 4.02 September 15, 2009 (available online at https://evs.nci.nih.gov/ftp1/ CTCAE/CTCAE_4.03/CTCAE_4.03_2010-06-14_QuickReference_8.5x11. pdf (accessed May 29, 2009)

19. Rab MAE, van Oirschot BA, Bos J, Kanne CK, Sheehan VA, van Beers EJ, et al. Characterization of sickling during controlled automated deoxygenation with oxygen gradient ektacytometry. J Vis Exp. (2019) 153:e60213. doi: 10.3791/60213

20. Rab MAE, van Oirschot BA, Bos J, Merkx TH, van Wesel ACW, Abdulmalik $\mathrm{O}$, et al. Rapid and reproducible characterization of sickling during automated deoxygenation in sickle cell disease patients. Am J Hematol. (2019) 94:57584. doi: 10.1002/ajh.25443

21. Rab MAE, Kanne CK, Bos J, Boisson C, van Oirschot BA, Nader E, et al. Methodological aspects of the oxygenoscan in sickle cell disease: a need for standardization. Am J Hematol. (2020) 95:E5-8. doi: 10.1002/ajh. 25655

22. Boisson C, Rab MAE, Nader E, Renoux C, Kanne C, Bos J, et al. Effects of genotypes and treatment on oxygenscan parameters in sickle cell disease. Cells. (2021) 10:811. doi: 10.3390/cells10040811

23. Biller E, Zhao Y, Berg M, Boggio L, Capocelli KE, Fang DC, et al. Red blood cell exchange in patients with sickle cell disease-indications and managements: a review and consensus report by therapeutic apheresis subsection of the AABB. Transfusion. (2018) 58:1965-72. doi: 10.1111/trf.14806

24. Billard M, Combet S, Hequet O, Kébaili K, Lorthois S, Pondarre C. Short-term femoral catheter insertion: a promising alternative to consistently allow longterm erythrocytapheresis therapy in children with sickle cell anemia. J Pediatr. (2013) 162:423-6. doi: 10.1016/j.jpeds.2012.09.009
25. Girard J, Tremisi PJ, Kassir A, Moullin T, Rigal D, Souillet G. Apheresis tolerance and acceptability in the child weighing $30 \mathrm{~kg}$ or less, with the exception of infants. Transf Clin Biol. (1996) 3:297-304. doi: 10.1016/S1246-7820(96)80020-6

26. Velasquez MP, Mariscalco MM, Golstein SL, Arewele GE. Erythrocytopheresis in children with sickle cell disease and acute chest syndrome. Pediatr Blood Cancer. (2009) 53:1060-3. doi: 10.1002/pbc.22211

27. Luzzi JR, Borba CC, Miyaji SC, Brito CA, Navarro-Xavier R, Dinardo CL. Reduced volume of red blood cell priming is safe for pediatric patients undergoing therapeutic plasma exchange. Transfus Apher Sci. (2021) 60:103005. doi: $10.1016 /$ j.transci.2020.103005

28. Swerdlow PS. Red cell exchange in sickle cell disease. Hematol Am Soc Hematol Educ Program. (2006) 2006:4853. doi: 10.1182/asheducation-2006.1.48

29. Jan K, Usami S, Sith JA. Effects on transfusion on rheological properties on blood sickle cell anemia. Transfusion. (1982) 22:17-20. doi: $10.1046 / j .1537-2995.1982 .2218215$ 4208.x

30. Quirolo K, Bertolone S, Hassell K, Howard T, King KE, Rhodes DK, et al. The evaluation of a new apheresis device for automated red blood cell exchange procedures in patients with sickle cell disease. Transfusion. (2015) 55:775-81. doi: 10.1111/trf.12891

31. Kim J, Joseph R, Matevosyan K, Sarode R. Comparison of Spectra Optia and COBE Spectra apheresis systems' performances for red blood cell exchange procedures. Transf Aph Sci. (2016) 55:368-70. doi: 10.1016/j.transci.2016.10.004

32. Aspner R, Schwarzenhofer M, Derfler K, Zauner C, Ratheiser K, Kranz A. Impairment of citrate metabolism in acute hepatic failure. Wien Klin Wochenschr. (1997) 109:123-7.

Conflict of Interest: The authors declare that the research was conducted in the absence of any commercial or financial relationships that could be construed as a potential conflict of interest.

Publisher's Note: All claims expressed in this article are solely those of the authors and do not necessarily represent those of their affiliated organizations, or those of the publisher, the editors and the reviewers. Any product that may be evaluated in this article, or claim that may be made by its manufacturer, is not guaranteed or endorsed by the publisher.

Copyright $\odot 2021$ Hequet, Boisson, Joly, Revesz, Kebaili, Gauthier, Renoux, Creppy, Nader, Nicolas, Berard, Cognasse, Vocanson, Bertrand and Connes. This is an openaccess article distributed under the terms of the Creative Commons Attribution License (CC BY). The use, distribution or reproduction in other forums is permitted, provided the original author(s) and the copyright owner(s) are credited and that the original publication in this journal is cited, in accordance with accepted academic practice. No use, distribution or reproduction is permitted which does not comply with these terms. 